Z. klin. Chem. u. klin. Biochem.

7. Jg., S. $467-473$, September 1969

\title{
Physikochemische, immunologische und biochemische Charakterisierung des Proteinanteils der low-density lipoproteins
}

\author{
Von D. ROELCKE und H. WeICKER \\ Aus dem Institut für Immunologie und Serologie der Universität Heidelberg (Direktor: Prof. Dr. E. Krab) \\ und der Abteilung für Stoffivechselforscbung der Medizinischen Universitäts-Poliklinik Heidelberg
}

(Leiter: Prof. Dr. H. Weicker)

(Eingegangen am 19. Mai 1969)

\begin{abstract}
Die Subfraktionierung von low-density lipoproteins (LDL) des Menschen an Ionenaustauschern wird beschrieben. Diese Methode wird der Ultrazentrifugation als Alternative zur präparativen Lipoprotein-Subfraktionierung gegenübergestellt. Das Verfahren trennt die LDL im Gegensatz zur Ultrazentrifugation auf Grund der Protein-Eigenschaften von LDL auf und darf deshalb als adäquat für immunologische Fragestellungen der LDL angesehen werden.

Antikörper gegen die 5 Subfraktionen zeigen alle LDL-Determinanten an, die mit Anti-LDL reagieren. Danach existieren keine immunologischen Unterschiede innerhalb der LDL-Grundantigenität. Im Gegensatz hierzu wird das gruppenspezifische $\mathrm{Lp}$ (a)-Merkmal nur auf einem Teil der LDL-Subfraktionen angetroffen. Das Lp(a)-Antigen ist Protein-gebunden, für die LDL-Grundantiginität ist offenbar der Lipid-Protein-Komplex verantwortlich.

Die 2-dimensionalen Aminosäurechromatogramme zeigen quantitative Unterschiede der Aminosäuren nach Hydrolyse der LDL-Fraktionen. Gleiche Bilder liefern Dünnschichtchromatogramme der delipidisierten LDL-Apoprotein-Subfraktionen. Der Triglycerid- und Cholesteringehalt der LDL und ihrer Subfraktionen ist höher als der Phosphatidgehalt. Das LDL-Apoprotein enthält geringe Mengen an Hexosen, die auf Cellulose-Dünnschichtplatten differenziert werden können.
\end{abstract}

\section{The physical-chemical, immunological and biochemical characterisation of the protein component of lon-density lipoproteins}

The practicability of subfractionating human low-density lipoproteins (LDL) by ion exchange chromatography is discussed. This method is considered as an alternative to the preparative subfractionation of lipoproteins by ultracentrifugation. Unlike ultracentrifugation, this method resolves LDL on the basis of the properties of the protein components, and it may therefore be suitable for the investigation of immunological problems.

Antibodies to the 5 subfractions show all the LDL determinants that react with anti-LDL. There are therefore no immunological variations within the basic LDL-antigenicity. The group-specific $\mathrm{Lp}(\mathrm{a})$ property, however, is only found in some of the LDL subfractions. The $\mathrm{Lp}(\mathrm{a})$ antigen is protein-linked, while the lipid-protein complex is evidently responsible for the basic LDL-antigenicity.

Two dimensional amino acid chromatograms show quantitative differences in the amino acids after hydrolysis of the LDL-subfractions. Identical results are obtained by the thin layer chromatography of the hydrolysed apoprotein from the lipid-free LDL-subfractions. In LDL and its subfractions, the concentration of triglycerides and cholesterol is higher than that of phosphatides. The LDL-apoprotein contains a small amount of hexoses, which can be differentiated on thin layer cellulose plates.

Die Entdeckung zahlreicher gruppenspezifischer Antigenean low-density lipoprotein-(LDL-)Molekülen ${ }^{1}$ ) hat der LDL-Forschung neue Aufgaben gestellt. Neben IsoAntikörpern, die in 1,2\% (DeLmas-Marsalet (1)) aller der Menschen, die häufig Bluttransfusionen erhielten, vorkommen und Antigene der großen $\mathrm{Ag}$-Gruppe anzeigen, ließen sich nach dem Modell LANDSTEINERs, durch Immunisierungs= und Absorptionsversuche gruppenspezifische (Erythrocyten-) Merkmale zu entdecken, auch Heteroantikörper induzieren, die mit dem gruppenspezifischen Merkmal Lp(a) (2) reagieren. Damit stellen die LDL ein immunologisch pluripotentes Material dar, das nur mit den ebenfalls allotypisch geprägten Immunglobulinen verglichen werden kann. Während bei den Immunglobulinen Proteinstrukturen als Antigene feststehen, muß bei den LDL-Antigenen erst entschieden werden, ob ihr relativ geringer Proteinanteil Träger der gruppenspezifischen Merkmale ist. Erste Untersuchungen über diese Frage haben gezeigt, daß für das Lp(a)-Merkmal die LDL-Proteinkomponente zuständig ist $(3,4)$.

1) $\mathrm{LDL}=\mathrm{Low}$-density lipoproteins $=\beta$-Lipoprotein, $\mathrm{HDL}=$ High - density lipoproteins $=\alpha(1)$-Lipoprotein.
Durch diesen Befund wird die immunologische LDLForschung auf das LDL-Apoprotein verwiesen, von dem sie ihren Ausgang nehmen muß.

Die LDL-Proteinkomponente ist bisher kaum charakterisiert worden - im Gegensatz zu den Lipidkomponenten. Untersuchungen über die endständigen Aminosäuren $\operatorname{der} \alpha_{2}$ - und $\beta$-Lipoproteine haben verschiedene Autoren vorgelegt (5-8), Bestimmungen der $\beta$ Lipoprotein-Aminosäuren-Zusammensetzung stammen von SHORE und SHORE (9). Erst seit den Arbeiten von Granda und SCANu (10) ist es möglich, das LDLProtein in löslicher Form darzustellen. Die Autoren haben ferner gezeigt, daß VeryLDL und $\mathrm{LDL}_{2}$ (wie $\mathrm{LDL}_{1}$ ) die gleiche Aminosäuren-Zusammensetzung haben, sich jedoch in den endständigen Aminosäuren unterscheiden.

Bei Chromatographieversuchen mit den LDL und ihrem isolierten Apoprotein haben wir eine (chromatographische) Heterogenität der LDL ermitteln können, die auf der LDL-Proteinheterogenität beruht (3). Diese neue Methode der Ionenaustauscher-Chromatographie, die LDL zu subfraktionieren, kann zur Charakterisierung der LDL-Proteine herangezogen werden. Sie un- 
terscheidet sich grundsätzlich von der Ultrazentrifugation und ist getade für immunologische Fragestellungen besonders geeignet.

Wir betrachten die AE-Cellulose-Chromatographie der LDL als eine neue Alternative zur Ultrazentrifugation und stellen deshalb in dieser Arbeit eine biochemische Analyse der AE-Subfraktionen vor.

\section{Material und Methoden}

\section{LDL-Darstellung}

LDL gewannen wir nach der Methode von HJERTEN (11) an Hydroxylapatit. Hydroxylapatit stellten wir nach den Angaben von Trselius und Mitarbeitern (12) her.

\section{LDL-Subfraktionierung und Apoprotein-Cbromatographie}

Die LDL-Subfraktionen bei $\mathrm{pH}$ 6,8 und die Apoprotein- und LDL-Subfraktionen bei pH 8,6/10,0 gewannen wir nach den Angaben von ROELCKE und Mitarbeitern (13) bzw. von ROELCKE und OsEI (3) an AE-Cellulose-Ionenaustauschern mit Phosphatund Tris-Puffern.

\section{LDL-Apoprotein-Darstellung}

Wir extrahierten die LDL mit Äther/Äthanol unter Dodecylsulfat-Zusatz nach den Angaben von GraNda und Scanu (10).

Anti-Lp (a) |-LDL

Anti-Ip (a) induzierten wir in Kaninchen durch i. v. Injektion von Ip $(a+)-L D L ~(14)$ und i. m. - Applikation der AE-Subfraktionen $3 \mathrm{AE}$ und $4 \mathrm{AE}$ (15).

\section{Oucbterlonj-Technik}

Wir bedienten uns der Makroausführung in Petrischalen. Neben Präzipitationsversuchen führten wir wegen der erhöhten Empfindlichkeit zusätzlich Präzipitations-Hemmtests aus (13).

\section{Biochemische Methoden}

Der Proteingehalt wurde nach Bestimmung des Stickstoffs mit der Kjeldahl-Methode errechnet. Die zweidimensionale Aminosäure-Chromatographie wurde nach den Angaben WeICKERs (16) auf Cellulose-Dünnschichtplatten ausgeführt. Die Gesamtlipidbestimmung wurde gravimetrisch ausgeführt (17). Die Phosphatide wurden nach Fiske und Subbarow (18) bestimmt. Das Cholesterin wurde nach der Methode von SchöNHEIMER und SPERRY (19) ermittelt. Die gesamten veresterten Fettsäuren wurden mit der Testpackung der Fa. Haury bestimmt $(20,21)$. Die chromatographische Darstellung der Lipide wurde auf KieselgelDünnschichtplatten mit dem Laufmittel Dichloräthan/Eisessig 100:1 ( $/ / v)$ vorgenommen (22). Die Fettsäuren wurden als Methylester gaschromatographisch ermittelt.

\section{Ergebnisse}

Physikochemische Untersuchungen

Zur Isolierung und Subfraktionierung der LDL wurde die Säulenchromatographie verwáandt. Die LDL wurden nach den Angaben von HJERTÉN (11) in Stufenelution mit Phosphatpuffern pH 6,8 an Hydroxylapatit dargestellt. Die Methode kann mit dem Verfahren der Gewinnung der Hydroxylapatitaffinen Proteine (23) kombiniert werden (Abb. 1). Die Hydroxylapatit-Fraktion $0,65 \mathrm{M}$ wurde von HJERTÉN (11) als $\beta$-Lipoprotein identifiziert. Cramér und Brattstein (24) haben gezeigt, $\mathrm{da} B$ es sich um die kompletten LDL handelt.

CRAMÉR und BRATTSTEIN gelang an Hydroxylapatit (24) keine Aufschlüsselung der LDL. Auch eine Subfraktionierung an Glasstaub ist nicht möglich (25). Versuche zur LDL-Subfraktionierung an anderen Adsorbentien oder mit Ionenaustauschern sind bisher nicht unternommen worden. Die Molekülsieb-Chromatographie an Sephadex führt nur eine Trennung der Lipoproteine in $\alpha$ - und $\beta$-Lipoproteine (HDL und LDL) ${ }^{1}$ ) herbei (26). Mit Phosphat-Puffern pH 6, 8 haben wir die LDL an AE-Cellulose-Ionenaustauschern in 4-5 Subfraktionen unterteilt ( $1 \mathrm{AE}-5 \mathrm{AE}$ ) (Abb. 2a). Diese Methode stellt neben der Ultrazentrifugation ein neues präparatives Verfahren zur LDL-Aufschlüsselung dar. Im Vergleich mit der Ultrazentrifugation sind folgende Gesichtspunkte hervorzuheben: Das Prinzip ist neu, die Methode kann ohne großen technischen Aufwand durchgeführt werden. Es wird bei den LDL normaler Seren absolute Reproduzierbarkeit angetroffen, da die Molaritätsdifferenz der Phosphatpuffer, die jeweils zur Desorption der einzelnen Subfraktionen führen, groß ist. Die Methode kann bei beliebigen Temperaturen angewandt werden. Der technische Aufwand ist bedeutend geringer als bei der Ultrazentrifugation:

Eine vom AE-Cellulose-Trenneffekt offenbar unabhängige LDL-Subfraktionierung gelang uns auch an DEAE-Cellulose-Austauschern (13) (Abb. 2b). Für den Ionenaustausch müssen (elektrostatisch) aktive Gruppen auf der Moleküloberfläche zugänglich sein. Dasselbe gilt für die Strukturen, die für die beim Ionenaus-

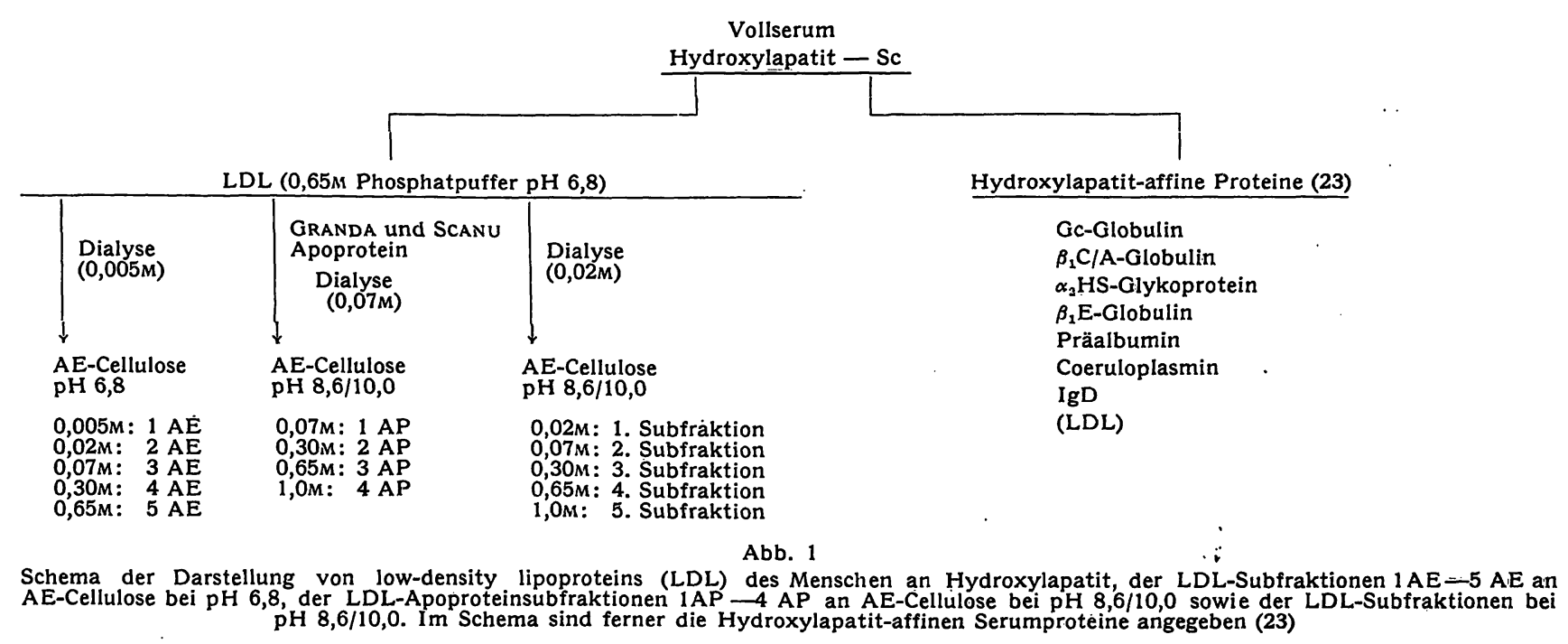



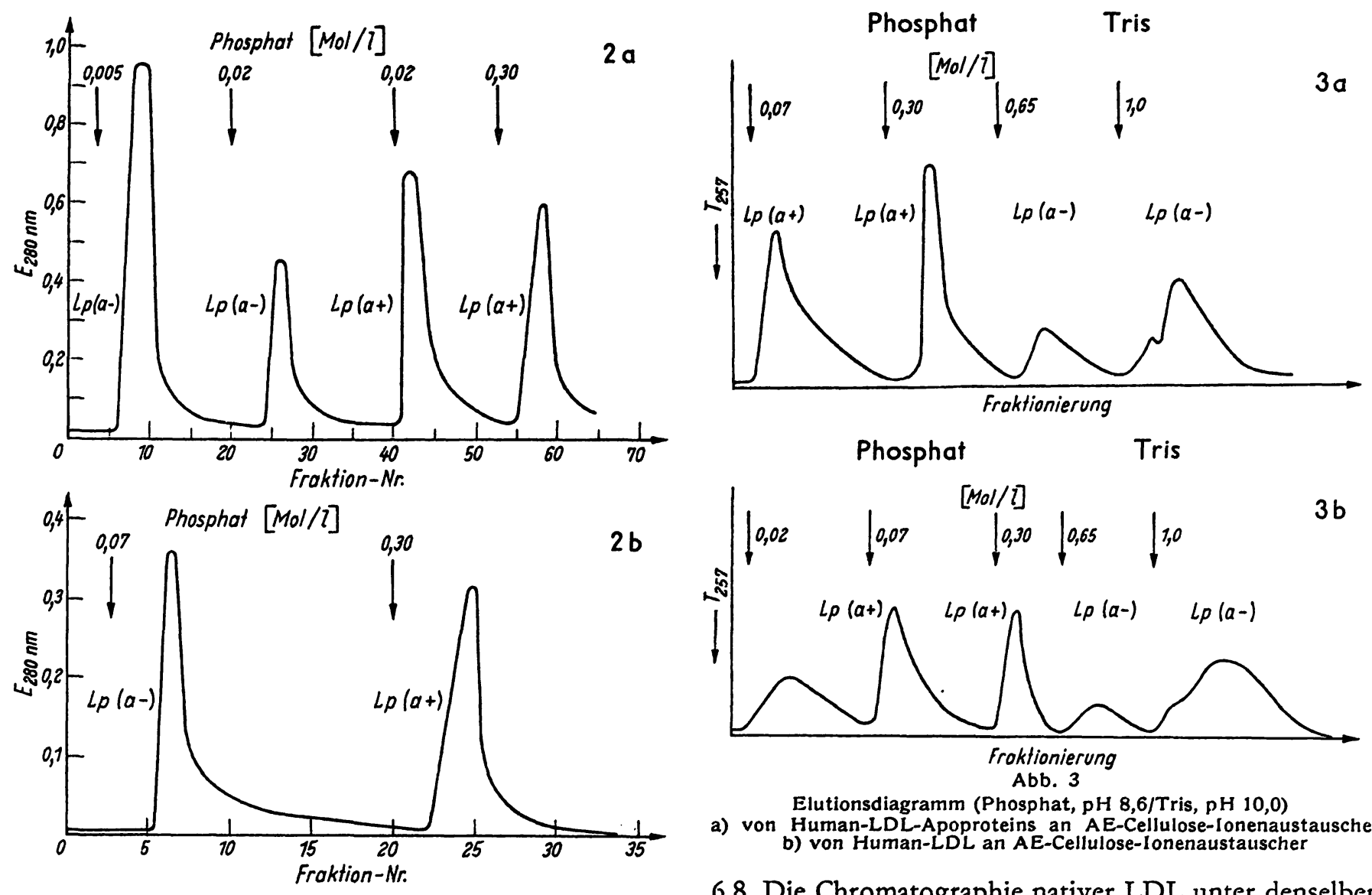

Elutionsdiagramm (Phosphat, $\mathrm{pH} 8,6 /$ Tris, $\mathrm{pH} \mathrm{10,0)}$

a) von Human-LDL-Apoproteins an AE-Cellulose-Ionenaustauscher b) von Human-LDL an AE-Cellulose-Ionenaustauscher

6,8. Die Chromatographie nativer LDL unter denselben Bedingungen führte jedoch $z u$ vergleichbaren Bildern (Abb. 3b). Wählt man 0,02 M Phosphat als ersten Puffer, so erhält man wie bei der Chromatographie bei pH 6,8 5 Gipfel, wobei Gipfel 2-5 sich ohne weiteres mit 1 AP bis 4 AP vergleichen lassen. Neben der Apoprotein-Heterogenität ist diesen Versuchen zu entnehmen, daß die Apoprotein-Heterogenität verantwortlich ist für die Subfraktionierbarkeit der LDL bei pH 8, 6/10,0. Vergleicht man die Chromatogramme nativer LDL, die mit $\mathrm{pH}$ 6,8- und $\mathrm{pH} 8,6 / 10,0$-Eluentien gewonnen wurden, so fällt auf, daß die Hauptkomponente einmal bereits mit dem ersten Puffer desorbiert ( $\mathrm{pH} 6,8$ ), bei pH 8,6/ 10,0 jedoch die höchste Affinität zu AE-Cellulose aufweist. Mit der Annahme, daß eine Umkehr im Desorptionsverhalten der einzelnen LDL-Subfraktionen stattgefunden hat, stehen auch die Höhen der entsprechenden Gipfel im Einklang. Eine derartige Inversion haben wir durch die LDL-Markierung mit dem immunologischen Merkmal Lp(a) sicherstellen können. Auf Grund dieser Befunde kann angenommen werden, $d a \beta$ auch die Chromatographie bei pH 6,8 die LDL-Proteinheterogenität widerspiegelt.

Immunologische Untersuchungen

Immunogene Strukturen müssen wie die Austauscheraktiven Gruppen in zugänglicher Form auf der Moleküloberfläche liegen. Deshalb lag es nahe, ein immunologisches Merkmal auf seinen Verbleib während der LDL-Chromatographie zu verfolgen. Wir wählten hierzu das Lp(a)-Merkmal, gegen das Hetero-Antikörper durch Immunisierung gewonnen werden können (Kaninchen). 
Tab. 1

Lp(a)-Aktivität der LDL- und LDL-Apoprotein-Subfraktionen, ermittelt in Präzipitations- und in Präzipitations-Hemm-Tests

\begin{tabular}{|c|c|c|c|c|c|c|}
\hline Untersuchtes Material & $0,005 \mathrm{M}$ & $\begin{array}{r}P \\
0,02 M \\
\end{array}$ & 0,07 & $\begin{array}{l}\text { Som } \\
\text { ivität }\end{array}$ & $0,65 \mathrm{M}$ & $\begin{array}{c}\text { Tris } \\
1,0 \mathrm{M} \mathrm{pH}_{10,0} \\
1\end{array}$ \\
\hline LDL, chromatographiert bei pH 6,8 & $\varnothing$ & $\varnothing$ & + & + & - & 一 \\
\hline LDL, chromatographiert bei pH $8,6 / 10,0$ & - & - & + & + & $\varnothing$ & $\varnothing$ \\
\hline $\begin{array}{l}\text { LDL-Apoprotein, chromatographiert bei } \\
\text { pH 8,6/10,0 }\end{array}$ & - & - & + & + & $\varnothing$ & $\bar{\sigma}$ \\
\hline
\end{tabular}

Der Lp(a)-Faktor ist innerhalb der 5 Subfraktionen der LDL, die bei pH 6,8 gewonnen werden können, auf die Subfraktionen $3 \mathrm{AE}$ und $4 \mathrm{AE}$ beschränkt. Die Hauptfraktion $1 \mathrm{AE}$ individueller $\mathrm{Lp}(\mathrm{a})$-positiver $\mathrm{LDL}$ ist $\mathrm{Lp}$ (a)-negativ, ebenso die Subfraktion $2 \mathrm{AE}$. Wir erhoben diese Befunde im Präzipitations- und im Präzipitationshemmtest. Durch die LDL-Chromatographie an AE-Cellulose bei pH-Werten von 8,6 (PhosphatPuffer) $/ 10,0$ (Tris) erhielten wir ebenfalls 5 Gipfel von denen der 2. und 3. Peak $\operatorname{Lp}(a)$ enthielten, während eindeutige Ergebnisse mit der 1 . Subfraktion $(0,02 \mathrm{MI})$ noch nicht erzielt werden konnten (Tab. 1). Vergleicht man die Chromatogramme bei pH 6,8 mit denen bei 8,6/10,0 im Zusammenhang mit der $\mathrm{Lp}(\mathrm{a})$-Aktivitāt der Gipfel, so ist ersichtlich, daß eine Umkehr im Desorptionsverhalten der einzelnen Fraktionen aufgetreten ist, bedingt durch den $\mathrm{pH}$-Wechsel. Als Ursache für diese $\mathrm{pH}$-abhängige Inversion kommen nur amphotere Moleküle in Betracht. Diese werden ausschließlich durch Aminosäuren gestellt, so daß auch die DesorptionsInversion nochmals unterstreicht, da $\beta$ der Proteinanteil der LDL für die chromatographische Trennung verantwortlich ist.

Das isolierte Apoprotein, chromatographiert bei $\mathrm{pH}$ 8,6/10,0, zeigt ebenfalls Lp(a)-Aktivität des $0,07 \mathrm{M}$ - und $0,30 \mathrm{nr}$-Eluates ( $1 \mathrm{AP}$ und $2 \mathrm{AP}$ ). Daraus geht hervor, daß - erwartungsgemäß - das volle LDL-Apoprotein

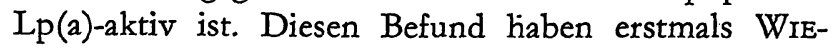
GANDT und Mitarbeiter (4) erhoben. Unsere Befunde bestätigen, daß die Lp(a)-Aktivität des Apoproteins gegenüber der der Ausgangs-LDL erhöht ist. Nimmt man das intakte $\mathrm{Lp}$ (a)-Merkmal als Maßstab für eine Alteration der LDL-Proteine während der Darstellung, so muß eine Denaturierung ausgeschlossen werden. Überraschend in diesem Zusammenhang ist die starke Einbuße der allgemeinen LDL-Heteroantigenität nach Delipidisierung.
Dieser Befund erlaubt die Annahme, daß Lipidstrukturen beim Aufbau dieser Heteroantigenität beteiligt sind.

Die Chromatogramme bei $\mathrm{pH} 6,8$ mit $\mathrm{Lp}(\mathrm{a})$-negativen LDL unterscheiden sich nicht von denen, die mit Lp(a)positiven LDL erzielt werden. Daraus folgt, daß die Lp(a)-Strukturen auf die LDL-Chromatographie keinen Einfluß nehmen. So kann angenommen werden, daß sie bei dem Protein-bedingten Austauscher-Proze $ß$ unbeteiligt sind, obwohl sie auf der Moleküloberfläche verankert sein müssen.

Die allgemeine LDL-Heteroantigenität ist einheitlich: Läßt man im OuchterLonYY-Test gegen die Subfraktionen 1-5 gerichtete Antikörper in Nachbarschaft von AntiLDL mit den LDL reagieren, so kommt es nicht zu Überkreuzungen oder Spornbildungen der Präzipitate, d. h. daß die $1 \mathrm{AE}$ bis $5 \mathrm{AE}$-Antikörper alle Determinanten der vollen LDL anzeigen, die Antigene $1 \mathrm{AE}$ bis $5 \mathrm{AE}$ also alle LDL-Partialantigene besitzen. Entsprechend kommt es auch bei Ansatz der einzelnen gegen die Subfraktionen gerichteten Antikörper untereinander gegen LDL nicht zu Spornbildungen (Abb. 4). Dieser Versuch wurde mit Antikörpern unternommen, die keine Anti-Lp(a)-Aktivität aufwiesen, um zu verhindern, daß durch Anti-Lp(a)-Komponenten der gegen $3 \mathrm{AE}$ und $4 \mathrm{AE}$ gerichteten Antikörper Differenzen in der Anti-LDL-Wirksamkeit der verschiedenen Antikörper vorgetäúscht werden. Nach diesen Befunden ist das Lp(a)-Merkmal das einzige LDL-Heteroantigen, das 1. gruppenspezifisch ist und 2. nur auf einem Teil der LDL-Subfraktionen vorkommt.

\section{Biochemische Untersuchungen}

Wie Tabelle 2 zeigt, unterscheidet sich der relative Proteingehalt der LDL und ihrer Subfraktionen. Er liegt niedriget in den $L p(a-)-S u b f r a k t i o n e n$ als in $\operatorname{den} L p(a+)$ -

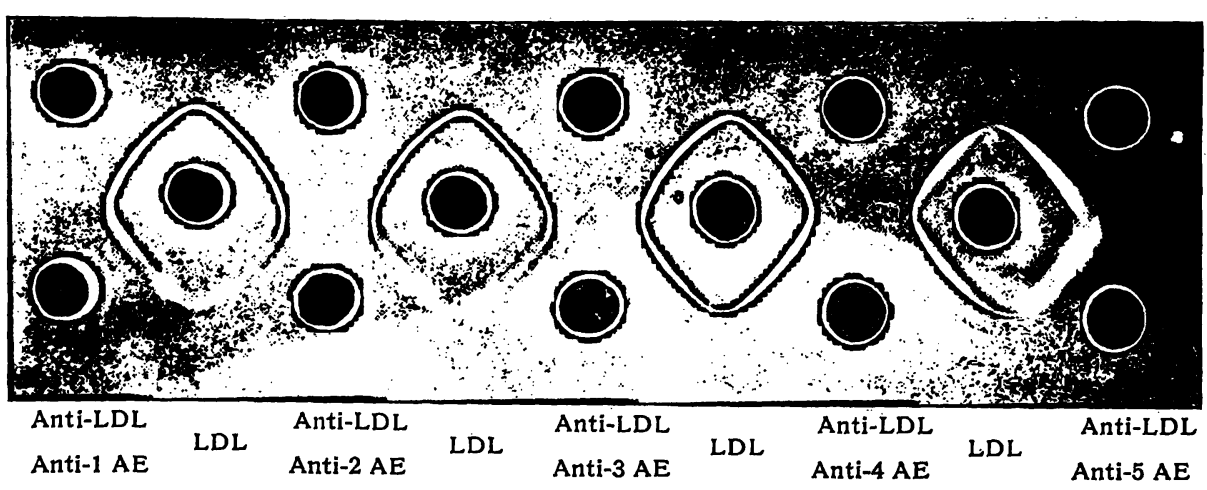

Abb. 4

Die Identität der Reaktion von AntiLDL mit der Reaktion der gegen die LDL-Subfraktionen $1 \mathrm{AE}-5 \mathrm{AE}$ gerichteten Antikörper 
Tab. 2

Protein- und Lipid-Gehalt von Human-Lp(a+)-LDL und inrer Subfraktionen $1 \mathrm{AE}-4 \mathrm{AE}$

\begin{tabular}{|c|c|c|c|c|c|}
\hline Material & Protein & $\begin{array}{l}\text { Gesamt- } \\
\text { Lipid } \\
\text { Gehal }\end{array}$ & $\begin{array}{l}\text { Cho- } \\
\text { lesterin } \\
\text { in \% der }\end{array}$ & $\begin{array}{l}\text { Phospha- } \\
\text { tide } \\
\text { LDL }\end{array}$ & $\begin{array}{l}\text { Trigly- } \\
\text { ceride }\end{array}$ \\
\hline $1 \mathrm{AE}$ & 19,6 & 80 & 26 & 17 & 37 \\
\hline $2 \mathrm{AE}$ & 23 & & 40 & 34 & - \\
\hline $3 \mathrm{AE}$ & 26 & 73 & 40 & 30 & - \\
\hline $4 \mathrm{AE}$ & 32 & 68 & 30 & 38 & - \\
\hline LDL & $(20-28)$ & $\begin{array}{c}75 \\
(72-80)\end{array}$ & 33 & 20 & 20 \\
\hline
\end{tabular}

Subfraktionen, die bei höheren Puffermolaritäten ( $\mathrm{pH}$ 6,8) desorbieren als der Lp(a-)-Hauptanteil und die ebenfalls Lp(a-)-LDL-Subfraktion 2 AE. Die absolute Proteinmenge der $\mathrm{Lp}(\mathrm{a})$-inaktiven Hauptsubfraktion war in den verschiedenen Versuchen jedoch regelmäßig mindestens doppelt so hoch wie die $\operatorname{der} \mathrm{Lp}(\mathrm{a})$-aktiven Hauptkomponente (4 AE), so daß die Lp(a)-Nachweisbarkeit nicht von der Konzentration eines immunologisch homogenen Proteinanteils der einzelnen Subfraktionen abhängen kann. Die Aminosäurenzusammensetzung der Voll-LDL und ihrer Subfraktionen unterscheidet sich quantitativ entsprechend dem relativen Proteinanteil der LDL und ihrer Subfraktionen (Abb. 5). Schwankungen des Gehaltes bestimmter Aminosäuren innerhalb der verschiedenen Subfraktionen werden in

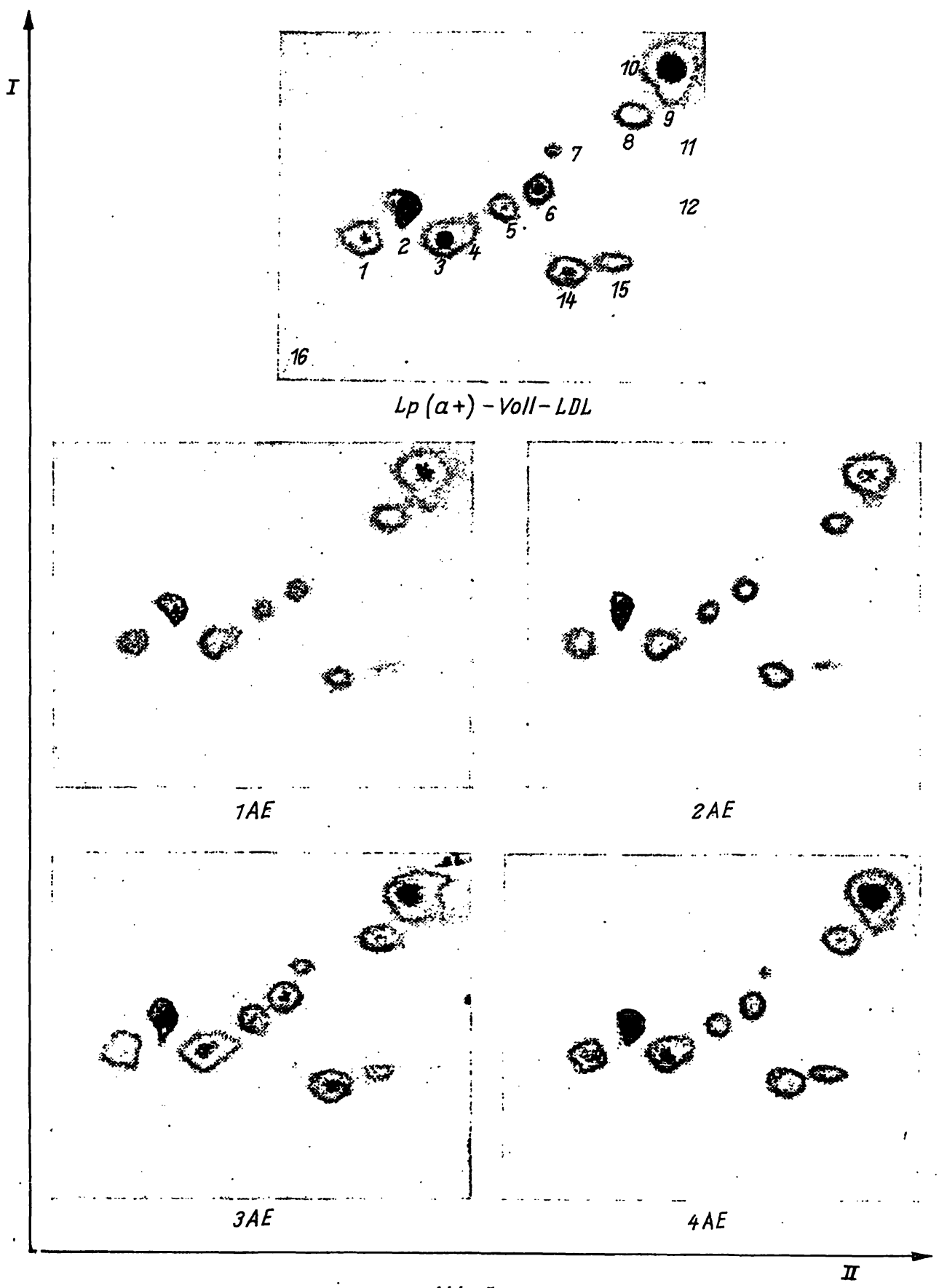

Abb. 5

Zweidimensionale Dünnschichtchromatographie der Aminosäuren der LDL und ihrer Subfraktionen auf Celluloseplatten nach Hydrolyse

Laufmittel: 1. Dimension: $n$ Butanol/Eisessig/Wasser $=12: 3: 5(\mathrm{v} / \mathrm{v}) ; 2$. Dimension: Phenol/Ammoniak/Wasser $=640: 3,4: 44(\mathrm{v} / \mathrm{v})$; . Darstellung mit 0,2proz. Ninhydrin in Aceton. Asparaginsaure, 2 Glutaminsaure, 3 Serin, 4 Glycin, 5 Threonin, 6 Alanin, 7 Tyrosin, 8 Valin, 9 Lelucin, 10 Phenylalanin, 11 Isoleucin, 12 Prolin, 13 Arginin, 14 Lysin, 15 Histidin. Bei 3 AE kommt auch Cystein (16) zur Darstellung. Zur Bezeich- 
der eindimensionalen Dünnschichtchromatographie sichtbar (Abb. 6). Hinweise auf die Biochemie des Lp(a)-Antigens können aus diesen Befunden nicht gewonnen werden.

In Tabelle 2 sind die Lipidwerte der LDL und ihrer Subfraktionen aufgeführt, die aus einem $\mathrm{Lp}$ (a)-positiven Serumpool gewonnen worden waren. Sie zeigen, daß mit Ausnahme der ersten Subfraktion nur sehr geringe Mengen von Triglyceriden nachweisbar sind. Der Cholesteringehalt nimmt kontinuierlich in den einzelnen Subfraktionen ab, die mit höheren Phosphatpuffermolaritäten eluiert worden waren. Hingegen steigt der Phosphatidgehalt in den $\mathrm{Lp}(\mathrm{a}+)$-Fraktionen an. Dünnschichtchromatogramme der Lipide bringt die Abbildung 7. Gaschromatographisch unterscheiden sich die Subfraktionen in ihrer Fettsäurezusammensetzung und lassen auf den Dünnschichtchromatogrammen unterschiedliche Konzentrationen der Lipidsubstanzen er-
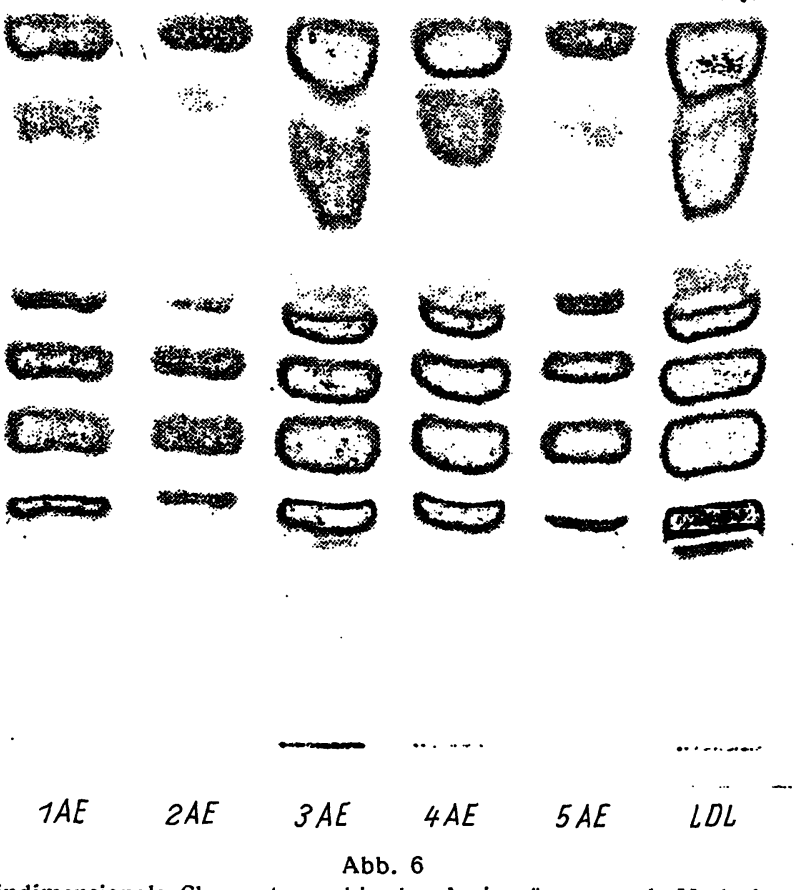

Eindimensionale Chromatographie der Aminosäuren nach Hydrolyse Dünnschichtplatten. Laufmittel: nPropanol/Eisessig/Wasser $=80$ : Dünnschichtplatten. Laufmittel: nPropar
$20: 20(\mathrm{v} / \mathrm{v})$
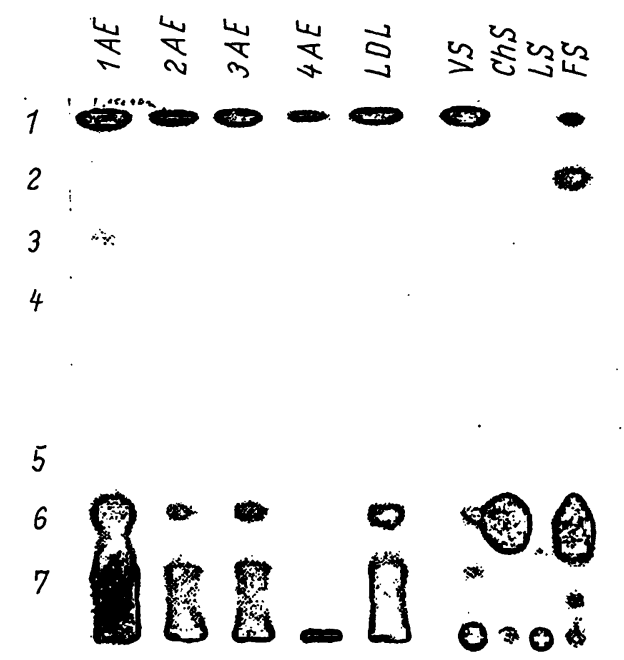

a)

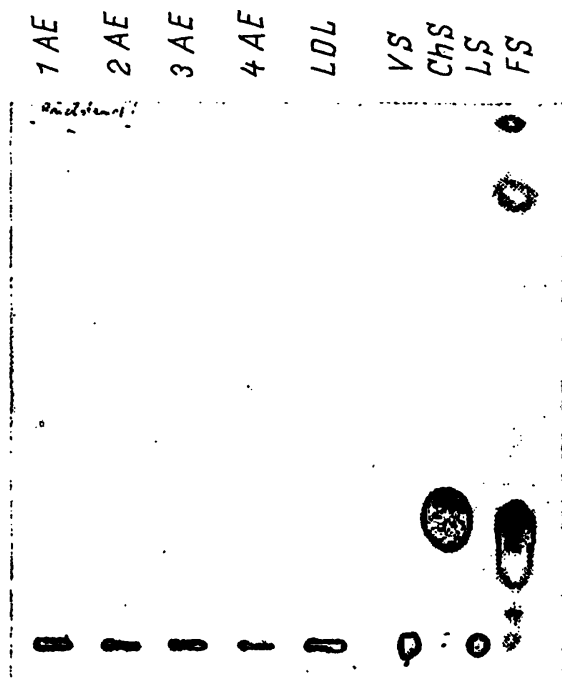

b) kennen. Einzelheiten bleiben einer weiteren Arbeit vorbehalten. Der Histidinfleck ist in den Chromatogrammen der LDL und ihrer Subfraktionen nur äußerst schwach ausgebildet.

Die Aminosäurenchromatogramme des Apoproteins und seiner Subfraktionen unterscheiden sich in ihrer $\mathrm{Zu}$ sammensetzung nicht bei den $\mathrm{Lp}(\mathrm{a}+)$ - und $\mathrm{Lp}\left(\mathrm{a}_{-}\right)$Subfraktionen sowie dem Apoprotein im Vergleich zu den entsprechenden Chromatogrammen der nativen LDL und ihrer Subfraktionen. Es bestehen ferner keine Unterschiede in der Aminosäurenzusammensetzung nativer $\mathrm{Lp}(\mathrm{a})$-aktiver und $\mathrm{Lp}$ (a)-inaktiver LDL und lediglich quantitative Unterschiede ihrer Subfraktionen $1 \mathrm{AE}$ bis $5 \mathrm{AE}$. Dieser Befund steht im Einklang mit dem säulenchromatographischen Verhalten $\mathrm{Lp}(\mathrm{a})$-positiver und $\mathrm{Lp}(\mathrm{a})$-negativer LDL, wobei ebenfalls keine Unterschiede festgestellt werden können.

In den LDL und ihrem Apoprotein können geringe Mengen an Kohlenhydraten nachgewiesen werden, die nach dünnschichtchromatographischen und kolorimetrischen Untersuchungen vorwiegend aus Hexosen bestehen.

\section{Diskussion}

Human-LDL können im präparativen Maßstab durch Chromatographie an AE-Cellulose mit Phosphat-Puffern $\mathrm{pH}$ 6,8 in 5 Subfraktionen aufgegliedert werden. Wie Chromatographieversuche mit dem isolierten LDLApoprotein und nativen LDL bei pH 8,6/10,0 zeigen, ist für die Subfraktionierung der Proteinanteil verantwortlich. $\mathrm{Da}$ die gruppenspezifischen immunologischen LDLMerkmale ebenso wie die Austauscher-aktiven Gruppen in frei zugänglicher Weise auf der Moleküloberfläche angeordnet sein müssen, sehen wir die LDL-Chromatographie als eine im Hinblick auf immunologische Fragen adäquate Methode der LDL-Aufschlüsselung an.

Das Verfahren unterscheidet sich grundsätzlich von der Ultrazentrifugation, da diese maßgeblich nach dem spezifischen Gewicht der LDL-Moleküle fraktioniert, das in erster Linie von der jeweiligen Lipidkonzentration abhängig ist. Daher ist die Ultrazentrifugation für $\mathrm{Li}$ piduntersuchungen der LDL die adäquate Methode, für

Abb. 7

Eindimensionale Chromatographie a) der acetonlöslichen Lipide, extrahiert aus den LDL und ihren Subfraktionen. Trägermaterial: KieselgelDünnschichtplatten, Laufmittel: Dichloräthan/Eisessig 100:1 (v/v) VS = Vollserum-Standard, ChS $=$ Cholesterin-Standard, LS = Lecithin-
Standard, FS = Fettsäuren-Standard, 1 Cholesterinester, 2, 3, 4, 5 Fettsäuren, 6 Cholesterin, 7 Triglyceride

b) der àcetonunlöslichen Lipide, s. Abbildung $7 \mathrm{a}$ 


\section{Lehrbuch}

\section{der Physiologischen Chemie}

von Prof. Dr. Franz Leuthardt

Begr. von Franz Edlbacher

15., neubearbeitete Auflage

mit 76 Abbildungen und 1 Bildtafel

Groß-Oktav. XVI, 912 Seiten. 1963.

Plastikeinband DM 42,-

Walter de Gruyter \& Co $\cdot$ Berlin

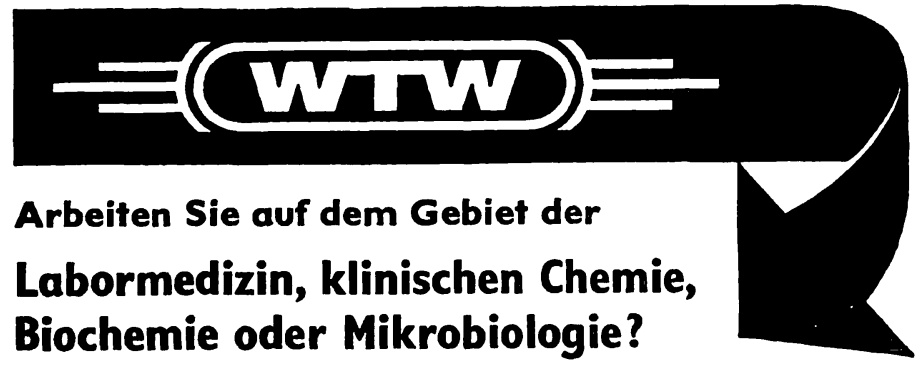

\section{WTW bietet Ihnen hierfür}

\section{Disc-Elektrophorese-Automat EA 100}

moderne Trennverfahren auf der Basis von Polyacrylamidgel mit hervorragenden Trenneigenschaften. Präparative und analytische Arbeitsweise, scharfe Fraktionstrennung, synchrone Steuerung durch einfache Programmierung, direktes Analysenergebnis. Ferner

\section{Thermoblock TB 4}

für Stickstoffbestimmung biologischer Substanzen, Bestimmung des gesamten und proteingebundenen Jod. Blutzuckerbestimmung nach der O-Toluidin-Methode, sowie

\section{Zählautomaten
für Bakterienkolonien.}

Einzelheiten durch Prospekt und ausführliche Literatur bei Ihrem Fachhändler oder direkt bei uns.

Wissenschatilich-Technische Werkstälten GmbH Dr.hab.K.Slevogt - 812 Weilheiin · Tel.(0881) 2638/2784

Verkaufbüros: Essen, Lönsberg 22,Tel. 53006

Hagen Hestertstraße 64 Tel 45857

Bad Nauheim. Frankfurter Str. 39, Tel. 4860

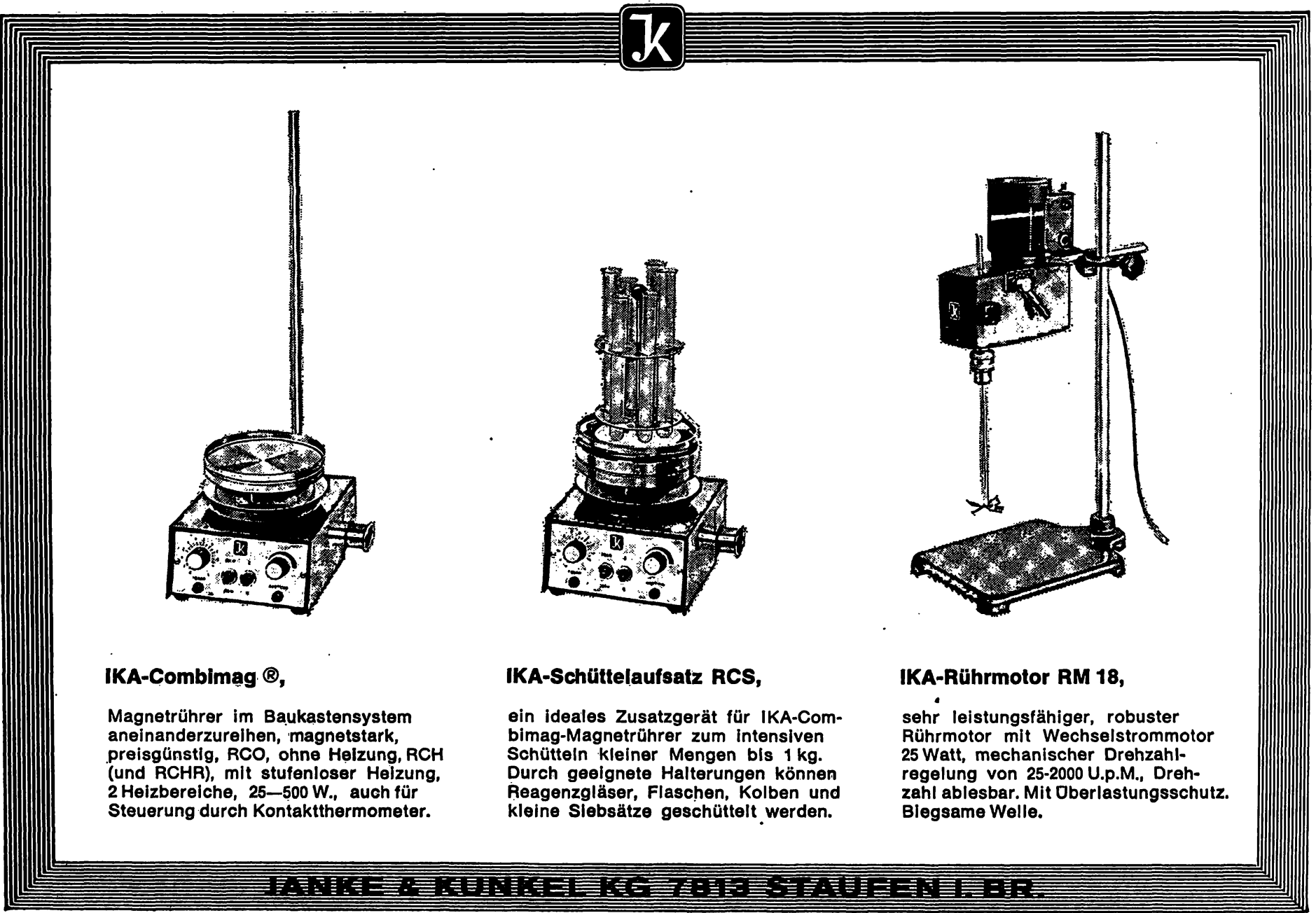




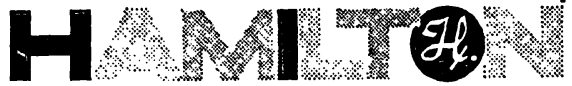

\section{für Präzision}

\section{und Qualität}

Passiert es Innen auch, das sich die Kanüle Ihrer Mikroliterspritze verstopft?

Diese Sorgen können Sie in Zukunft vergessen!

Wir empfehlen Ihnen den HAMILTON-SPRITZENREINIGER*

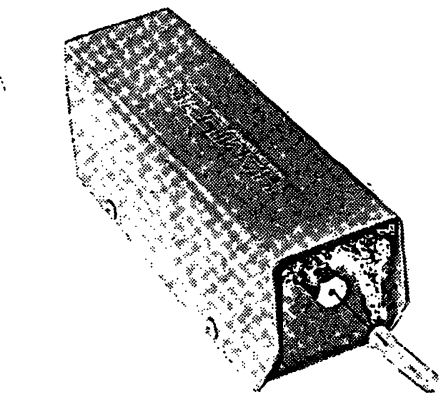

*DM 275.-

zur gründlichen Reinigung von Mikroliterspritzen aller Fabrikate.

Fragen Sie Ihren

HAMILTON - FACHBERATER

Generalagentur für Europa:

\section{MICROMESURE N.V.}

Postfach 205, DEN HAAG Holland

Autorisierter Händler für die BRD:

\section{GUNTHER SCHMIDT}

2 Hamburg 68, Postfach 680104

Bezirksvertretungen:

$\begin{array}{ll}\text { Aachen: } & \text { Fa. Ludwig Mohren OHG } \\ \text { Berlin: } & \text { Fa. K. Marggraf } \\ \text { Bonn: } & \text { Fa. C. Gerhardt } \\ \text { Bremen: } & \text { Fa. H. Jürgens \& Co. } \\ \text { Frankfurt: } & \text { Fa. Willi Fischer \& Co. } \\ \text { Freiburg: } & \text { Fa. Bender \& Hobein GmbH } \\ \text { Göttingen: } & \text { Fa. Bodo Schmidt } \\ \text { Hannover: } & \text { Fa. H. Jürgens \& Co. } \\ \text { Karlsruhe: } & \text { Fa. Bender \& Hobein } \\ \text { Kiel: } & \text { Fa. Erich Eydam } \\ \text { München: } & \text { Fa. Bender \& Hobein GmbH } \\ \text { Münster: } & \text { Fa. H. Jürgens \& Co. }\end{array}$
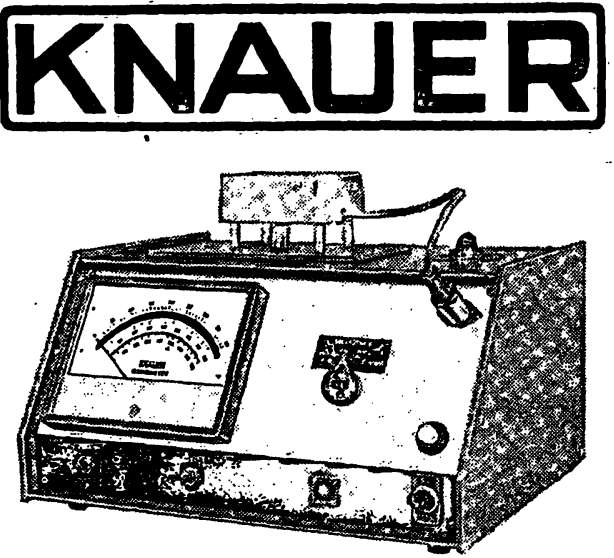

\section{ELEKTRONISCHES HALBMIKRO-OSMOMETER}

zur direkten Bestimmung der Os molalität aller Körperflüssigkeiten wie Blut, Serum, Urin, Liquor durch Gefrierpunktmessung.

- Probevolumen nur $0,15 \mathrm{ml}$ oder $0,05 \mathrm{ml}$

- Messdauer ca. 2 Minuten

- Genauigkeit und Reproduzierbarkeit 1-2 Millios$\mathrm{mol} / \mathrm{kg}$ bzw. $1 \%$

- Preis DM 3600,- + Mehrwertstever

- Lieferung kurzfristig ab Lager

Weitere Spezialität: Komplettes System zur Molekulargewichtsbestimmung zwischen 100 und 1000000 durch Kryoskopie, Dampfdruck-Osmometrie und Membran-Osmometrie.

Wissenschaftlicher Gerätebau

KG Dr. Ing. Herbert Knauer \& Co. GmbH

1 Berlin 37 (West) - Holstweg 18 - Telefon 848705

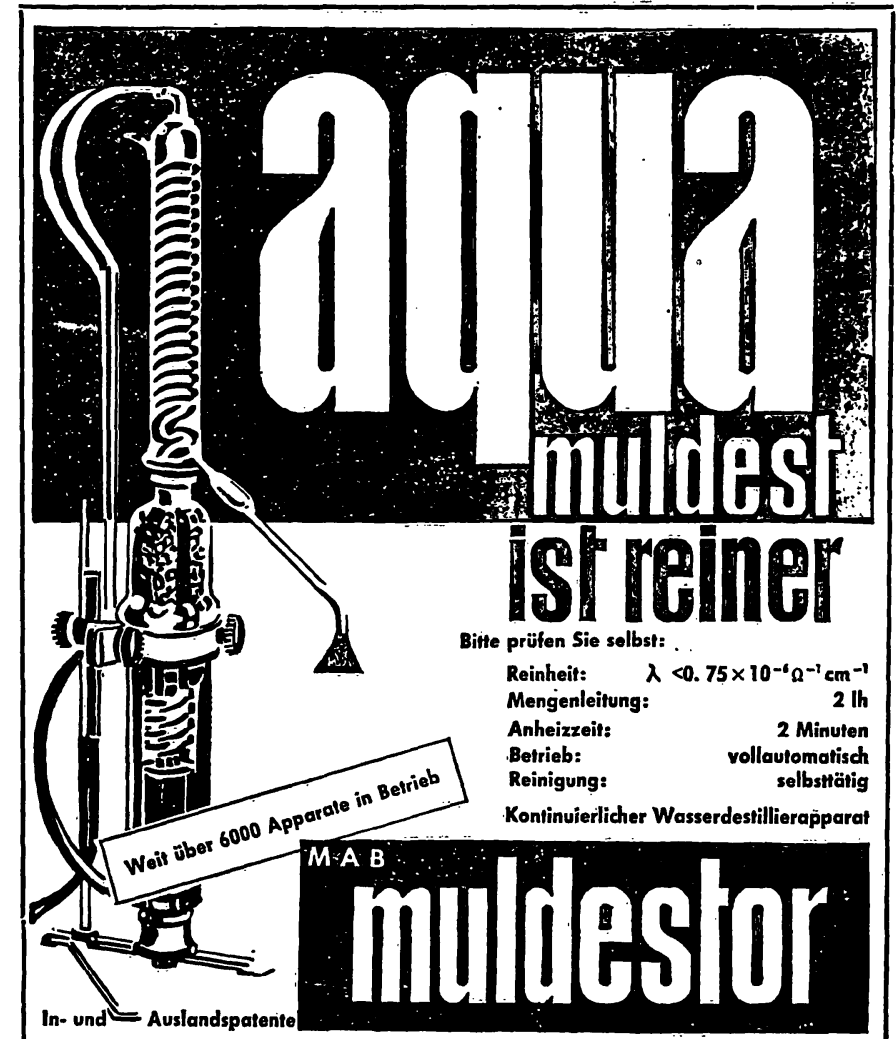

Lieferung durch: WAGNER \& MUNZ Laboratoriums-Bedarf

8 München 2, Luisenstr. 25, Tel. 594023 oder über den Fachhandel 
immunologische Fragestellungen erscheint sie jedoch weniger geeignet.

Die Chromatographie kann auch nicht durch die Elektrophorese ersetzt werden. Präparative Elektrophoresen sind auf spezialisierte Laboratorien angewiesen. Neben der Netto-Ladung, die eine Funktion der Moleküloberfläche ist, interveniert bei der Elektrophorese jedoch auch die Molekülgröße, die wiederum durch den hohen und zudem stark variierenden Lipidgehalt bestimmt ist. Darin unterscheidet sich auch die Elektrophorese grundsätzlich von der Chromatographie. Als Ausdruck des Molekülgrößen-Spektrums tritt in der Papierelektrophorese ein starkes tailing $\operatorname{der} \beta$-Lipoproteine (=LDL) auf, und bei der Discelektrophorese in üblicher Technik bleiben die LDL wegen ihres hohen Molekulargewichtes am Startpunkt liegen. Auch mit modifizierter Technik werden mit der Discelektrophorese keine eindeutigen Befunde erhoben (4). Eine Auftrennung der LDL in $\beta$-Lipoprotein und $\alpha_{2}$-Lipoprotein in der Stärkegelelektrophorese ist möglich, in Agar als Medium haben HoutsMULLER und Mitarbeiter (27) ebenfalls 2 Fraktionen beobachtet, doch läßt sich bei diesen Versuchen nicht abschätzen, ob bei der Subfraktionierung der Protein- oder der Lipidanteil dominiert. Wir sahen in der Immunelektrophorese in Agar eine konzentrationsabhängige extreme Variabilität der LDL-Wanderungsgeschwindigkeit (von $\beta_{1}$ - bis in Albuminposition). Dieser Befund bestätigt die von Grabar und Burtin getroffenen Feststellungen (28).

Die Austauscher-Chromatographie kann also als eine neue Alternative zur Ultrazentrifugation empfohlen werden. Wir haben deshalb neben den vollen LDL die Subfraktionen (bei $\mathrm{pH}$ 6,8) biochemisch untersucht. Die biochemischen Daten der Tabelle 2 stützen sich auf die LDL und ihre Subfraktionen von gepoolten Seren mehrerer Spender. Sie dürfen daher als repräsentative Werte angesehen werden. In Versuchen mit den Subfraktionen einzelner Spender sahen wir teilweise abweichende Lipid-Befunde. Sie können auf die situationsbedingte $\mathrm{Li}$ - pidbeladung der Proteine zurückgeführt werden, die durch die entsprechende Stoffwechsellage verursacht wird. Hierfür sprechen auch Unterschiede in den Befunden, die mit den Subfraktionen eines Spenders bei verschiedenen Blutentnahmen erhoben wurden.

Im Gegensatz zu den Lipiden verhält sich die relative Proteinmenge in den verschiedenen Versuchen einheitlich. Charakteristisch ist die Zunahme des Proteinanteils in den Subfraktionen, die mit steigenden Puffermolaritäten eluiert werden. Dieses Verhalten spiegelt sich in den Befunden der Aminosäurenzusammensetzung der einzelnen Subfraktionen wider, indem sich die Aminosäurenzusammensetzung der Voll-LDL und ihrer Subfraktionen lediglich quantitativ unterscheidet. Im $\mathrm{Zu}$ sammenhang mit den $\mathrm{Lp}(\mathrm{a})$-Bestimmungen zeigen diese Befunde, da $\mathrm{B}$ sich die entsprechenden Subfraktionen verschiedener Versuche hinsichtlich ihres Proteinanteils gleichen. Diese Ergebnisse stehen in Einklang mit der Reproduzierbarkeit der Ionenaustausch-LDL-Subfraktionierungsmethode, die auf dem Proteinanteil basiert. Während die Bestimmungen der Aminosäuren und der Lipide in qualitativer Hinsicht keine für die einzelnen Subfraktionen charakteristischen bzw. konstanten Bilder ergeben, kann eine Kennzeichnung der Subfraktionen auf immunologischem Wege erreicht werden. So kann beispielsweise das Lp(a)-Merkmal geradezu als "marker" für die LDL-Subfraktionen dienen. Inwieweit andere immunologische Merkmale diesen Zweck erfüllen können, wird in weiteren Experimenten ermittelt werden. Erste Untersuchungen in dieser Richtung haben gezeigt, daß die LDL-Hauptsubfraktion $1 \mathrm{AE}(\mathrm{pH} 6,8)$ im Gegensatz zu Lp(a) Merkmalsträger für die $\mathrm{Ag}(\mathrm{x})$ - und $\mathrm{Ag}(\mathrm{e})$ Faktoren ist (29).

$\mathrm{Ob}$ bei den Determinanten der Lp- und Ag-Faktoren prosthetische Kohlenhydratgruppen von Bedeutung sind, kann bei dem gegenwärtigen Stand der Untersuchungen noch nicht entschieden werden. Wir haben bisher durch Neuraminidase keine Lp(a)-Rezeptor-Inaktivierung herbeiführen können.

\section{Literatur}

1. Delmas-Marsalet, Y. und M. Goudemand, Vox Sang. 14, 179 (1968). - 2. BerG, K., Acta path. microbiol. Scand. 59, 369 (1963). - 3. Roexcke, D. und C. Oser, Immunology (im Druck). 4. WIEgandt, H., K. Lipp und G. G. Wendt, Hoppe-Seyler's Z. physiol. Chem., 349, 489 (1968). - 5. Brown, R. K., R. E. Davis, B. Clark und H. van Vunakis, The blood lipids and the clearing Factor, S. 104 (III. Intern. Conf. Biochem. Problems of Lipids), Koninklijke Vlaamse Academie voor Wetenschappen, Letteren en Schone Kunsten von Belgie. Paleis der Academien, Brussels, (1956) 6. Lindgren, F. R. und A. V. Nrchols, Structure and Function of Human Serum Lipoproteins, in F.W. Putnam (Ed.), The Plasma Proteins, Vol. II, S. 1, Academic Press, New York (1960). 7. Robdell, M., Science, Washington 127, 701 (1958). - 8. ShORE, B., Arch. Biochem. Biophysics 71, 1 (1957). - 9. SHORE, B. und V. G. Shore, Plasma (Milan) 2, 621 (1954). - 10. Granda, J. L. und A. ScANU, Biochemistry (USA) 5, 3301 (1966). - 11. HJERTÉN, S., Biochim. biophysica Acta 31, 216 (1959). - 12. Tiselius, A., S. HJertén und O. Levin, Arch. Biochem. Biophysiç 65, 132 (1956). - 13. Roelcke, D., E. KRẠ und A. Fafimian, Blut 18, 160 (1968). - 14. BerG, K. und G. G. WendT, Humangenetik 1, 24 (1964). - 15. RozLCKe, D. und E. KRAH (in Vorbereitung).
16. Weicker, H., diese Z. 6, 221 (1968). - 17. KunKel, H. G., H. E. Ahreins und W. J. Eisenmenger, Gastroenterology 11, 499 (1948). - 18. Fiske, Ch. und Y. Subbarow, J. biol. Chemistry 66, 375 (1925). - 19. SchöNHEIMER, R. und W. M. SPERRY, J. biol. Chemistry 106, 745 (1942). - 20. Chabrol, E., M. BöszorMéNYI und P. FALLOT, Sem. hôp. Paris 25, 3446 (1949). - 21. Fried, R. und J. Hoeflmayr, Klin. Wschr. 41, 727 (1963). 22. SperRY, W. M., Lipidanalysis, in D. Glick: Methods of Biochemical Analysis, Vol. II, S. 83, Interscience Publ. New York, London (1955). - 23. RoelCKe, D. und U. BoDEM (in Vorbereitưng). - 24. CRAMÉr, K. und J. Brattstein, J. Atheroscler. Res. 1, 335 (1961). - 25. Carlson, L. A., Scand. J. Clin. Laborat. Invest. 6, 70 (1954). - 26. Franzinr, C., Clin. chim. Acta Amsterdam 14, 576 (1966). - 27. Houtsmuller, A. J., A. HuyssonHaAsdyk, A. Huysman und E. Rinkel-van Driel, Clin. chim. Acta Amsterdam 9, 497 (1964). - 28. Grabar, P. und P. Burtin, Immunelektrophoretische Analyse, S. 138-141, bearb. von G. Hermann, Elsevier Publ., Amsterdam (1964). - 29. Roelcke, D. und R. BütLER (unveröffentl. Befunde).

Dr. D. Roelcke, 6900 Heidelberg Voßstraße 2

Prof. Dr. H. Weicker 6900 Heidelberg Hospitalstraße 